\title{
Changing pattern of pathogens and their antibiogram in pyogenic meningitis - a two years study from India
}

\begin{abstract}
A 2 years clinico-laboratory study was conducted at one of the premium tertiary care Institute in India. Objective of the study was to see if there is any change in existing Microbial profile and their antibiogram in admitted cases of acute bacterial meningitis. A definitive change in bacterial profile and antibiogram was noted along with change in seasonal variation of acute bacterial meningitis (ABM). Acinetobacter and Gram negative organisms were found to be increasing in numbers in comparison to Gram positive organisms in Children. Whereas in adults Gram Positive organisms were found to be major pathogens but their sensitivity patter changed with increased resistance to penicillin. This change in bacterial profile and antibiogram is very important to assess and communicate to clinicians for better, early and effective management of ABM patients.
\end{abstract}

Volume 8 Issue 3 - 2020

\section{Dimple Kasana \\ Department of Microbiology, Guru Gobind Singh Indraprastha University, India}

Correspondence: Dimple Kasana,Vardhman Mahavir medical college and Safdarjang Hospital, Guru Gobind Singh Indraprastha University, New Delhi, India, Tel 9990206500, Fax 01126165060 , Emaildimplekaana@gmail.com

Received: July 31, 2020 | Published: August 18, 2020

Keywords: bacterial meningitis, antibiogram, pathogens, cerebrospinal fluid, gram negative organisms, microbial profile

\section{Introduction}

Bacterial meningitis is a medical emergency associated with high mortality rates. Cerebrospinal fluid (CSF) culture is the "gold standard" for diagnosis of meningitis and it is important to establish the susceptibility of the causative microorganism to rationalize treatment. Disease prognosis depends on a number of factors like age of the patient, etiological agent, time gap between onset of symptoms and first dose of antibiotic, immunization status and geographical distribution of the agent. In most cases, delay of start of antibiotic is associated with increased rate of neurological sequel as well as increased rate of mortality and morbidity associated with meningitis. Hence, in majority of cases antimicrobial therapy is started on the basis of presumptive treatment which is based on the etiology and antibiotic susceptibility pattern of the most common pathogens prevalent .Hence, knowledge of most common etiological agent and their antibiogram pattern can help in deciding most effective drug treatments to be given to the patients. It is important to start empirical therapy in Acute bacterial meningitis as it's a Medical emergency. It is therefore important to know the current existing microbial profile and their antibiogram pattern and if it's changing in pattern then previous years. Our study would identify if pattern has changed in recent years and hence shall be useful in deciding empirical urgent therapy for the patient before culture sensitivity reports are available.

\section{Objectives}

The objective of this study was:

I. To assess the emerging pathogens and antimicrobial sensitivity patterns in cases of clinically suspected acute bacterial meningitis.

II. To identify the newer or changing microbial and antibiogram pattern if any.

\section{Materials and methods}

CSF sample was processed as per standard protocol and isolation, identification and antimicrobial susceptibility pattern was determined. Our study showed most susceptible age group was 1-6 months $(52.3 \%$ cases) with $72 \%$ of cases under one year of age. There was male preponderance with $\mathrm{M} ; \mathrm{F}$ ratio of 1.6:1, showing younger age and male sex were more susceptible. This is similar to finding reported by various studies. All cerebrospinal fluid samples were macroscopically examined for turbidity, hemorrhagic CSF and cobweb appearance. Direct microscopy by doing Gram's stain was done for pus cells, red blood cells and micro-organisms. Culture was put on chocolate agar, blood agar and Mac Conkey agar in the Co2 Incubator at 37degree Celsius for 48 hours. The rest samples were incubated in the brain heart infusion broth for 48 hours. A battery of biochemical and serological tests were done for identification of the organism. Our study included 4500 cases of suspected meningitis in children and adults admitted in pediatric and medicine wards /ICU from June 2017 to June 2019.

\section{Results and discussion}

A total number of 4500 CSF (Cerebro Spinal Fluid) samples were received at Department of Microbiology in a 2 years study period (June2017-2019). 22\% (950) of sample showed growth. We have received more adult samples than pediatrics and the ratio of adult: pediatric was $5: 1$. It could be because our Nursery patient's i.e. (0- 1 year of age) samples were processed separately and were not included in this study for the sake of convenience. Maximum number of cases (534) was seen during September-December. In pediatric population the commonest organism was Acinetobacter (35\%) followed by Staphylococcus aureus (30\%), a Streptococcus (20\%), Pneumococcus, Eschirichia coli, Klebsiella pneumoniae and Haemophilus influenza (10\%). This picture was different than the earlier expected results of more GPC than GNB as commonest cause 
in admitted Pediatric population and Acinetobacter was surprisingly emerged as the commonest organism. Emergence of Acinetobacter spp. as an important cause of meningitis has now being increasingly reported and various studies have now reported gram negative as more commonly isolated organism as compared to gram positive organism and increased rate of isolation of Acinetobacter spp. Staphylococcus aureus $(30 \%)$ was the commonest organism in the adult population. Amongst Gram negative isolates, maximum were sensitive to Cefoperazone + salbactum $(91 \%)$ followed by imipenem $(85 \%)$, piperacillin tazobactum (78\%), meropenem (71\%) and netilmicillin, amikacin and ceftazidime (50\%). In Gram positive organisms all were sensitive to Vancomycin, Chloramphenicol followed by Clindamycin (70\%), Erythromycin (64\%), Gentamicin (55\%), Ciprofloxacin and Penicillin showed least sensitivity of $41 \%$ only.

\section{Discussion and conclusions}

Acinetobacter is an emerging pathogen in the pediatric age group. Staphylococcus aureus was the commonest organism in the adult population. The resistance of the common isolates to penicillin is high. Cefoperazone salbactum combination, Carbapenems (e.g., meropenem) and piperacillin-tazobactam should be considered for treating severely ill patients. Thus, our study concludes that most effective drugs against gram positive are clindamycin, vancomycin and linezolid whereas for gram negative most effective antibiotics are netilmicin, imipenem and colistin. A new trend in drug resistance was noted, i.e. there is an increased development for resistance against ciprofloxacin both in gram positive and gram- negative organisms. Thus, our study shares some insight for the most common emerging and changing pattern of organism associated with meningitis in children and adults indoor patients and also most effective drugs to help the clinician to provide most effective antimicrobial therapy both in presumptive as well as targeted approach of treatment and hence decreasing significant mortality, morbidity and long term sequel associated with meningitis. Such microbiological studies should be continued with regular periodicity for keeping an active eye and surveillance on change, if any, in microbial profile and antibiogram of the causative organisms. It would help clinician in better and proactive management of the acute bacterial meningitis Patients.

\section{Acknowledgments}

None.

\section{Conflicts of interest}

The author declares that they have no conflicts of interest.

\section{References}

1. Oordt-Speets AM, Bolijn R, Van Hoorn RC, et al. Global etiology of bacterial meningitis: A systematic review and meta-analysis. PLoS One. 2018;13(6):e0198772.

2. Leazer R, Erickson N, Paulson J, et al. Epidemiology of Cerebrospinal Fluid Cultures and Time to Detection in Term Infants. Pediatrics. 2017;139(5):e20163268.

3. Assegu Fenta D, Lemma K, Tadele H, et al. Antimicrobial sensitivity profile and bacterial isolates among suspected pyogenic meningitis patients attending at Hawassa University Hospital: Cross-sectional study. BMC Microbiol. 2020;20(1):125.

4. Viallon A, Botelho-Nevers E, Zeni F. Clinical decision rules for acute bacterial meningitis: current insights. Open Access Emerg Med. 2016;8:716.

5. Russell FM, Biribo SS, Selvaraj G, et al. As a bacterial culture medium, citrated sheep blood agar is a practical alternative to citrated human blood agar in laboratories of developing countries. J Clin Microbiol. 2006;44(9):3346-3351.

6. Laboratory Methods for the Diagnosis of Meningitis caused by Neisseria meningitidis, Streptococcus pneumoniae, and Haemophilus influenza. 